Conversely, derivatives up to the order $f^{(m)}(x)$ may be obtained from Eq. (6) by termwise differentiation. In the expressions for $f(x)$ and any derivative up to $f^{(m-1)}(x)$, any sine series represents a function having the value zero at $x= \pm a$, thus facilitating evaluation at these points. In some solutions Eq. (5) is a convenient expression for $f^{(m)}(x)$. If desired, Eq. (5) can be modified by adding a term $c^{\prime \prime} x$ combined with $\sum_{1}^{\infty}$ $b_{n}^{\prime} \sin \beta_{n} x$ so that the sine series in this equation also represents a function having the value zero at $x= \pm a$. Then Eq. (6), modified by this additional term, is

$$
f(x)=\sum_{0}^{m+1} c_{n} x^{n}+\sum_{1}^{\infty}\left(a_{n} \cos \beta_{n} x+b_{n} \sin \beta_{n} x\right) .
$$

Equations (6) and (6a) are special cases of Borel's theorem ${ }^{1}$ which apply to derivatives of any finite order up to $f^{(m)}(x)$.

The preceding methods of derivation, stated in terms of a single variable $x$, also apply completely to a three-dimensional member bounded by parallel planes $x= \pm a$ with assigned boundary conditions. In this case $f(x)$ is replaced by $f(x, y, z)$ and the derivatives are written as partial derivatives with respect to $x, f^{(m)}(x)$ becoming $\partial^{m} f / \partial x^{m}$. Also in Eqs. (1a), (2a), (6), (6a), every coefficient $a_{n}, b_{n}, c_{n}, c$, while independent of $x$, is a function of $y$ and $z$. For example, in Eq. (1a), $b_{n}$ is $b_{n}(y, z)$, and $c$ is $c(y, z)=$ $f(a, y, z) / a$. Thus $f(x, y, z)$ and each of its $x$-derivatives of finite order can be expressed as the sum of a finite power series and a Fourier series without discontinuity at $x= \pm a$; the expressions can, therefore, be evaluated definitely at these boundaries.

${ }^{1} \mathrm{E}$. Borel, Lȩons sur les fonctions de variables réelles, Gauthier-Villars, Paris, 1905, p. 68.

\title{
A TAPERED LINE TERMINATION AT MICROWAVES*
}

\section{By GEORGE J. CLEMENS (City College of New York)}

1. Introduction. In the field of ultra-high frequency, one method of power transmission is by means of concentric metallic conductors called a co-axial transmission line. The maximum amount of power can be delivered over these lines if the impedance of the load is equal to the characteristic impedance ${ }^{1}$ of the line. In addition, in various measurements at ultra-high frequency it is essential to have a matched termination over a broad band of frequencies. This problem was approached experimentally and led to the tapered line termination as shown in Fig. 1.

To the left of $x=0$, the co-axial line has an inner metallic conductor of radius $a$. From $x=0$ to $x=L$, the inner conductor is a glass tube coated with a thin metallic film of resistive material. ${ }^{2}$ To the left of $x=0$, the outer conductor has a radius $b$ and from $x=0$ to $x=L$, the outer conductor has a linear taper down to a radius $c$. Also at $x=L$, the inner and outer conductors are short circuited.

*Received Dec. 2, 1948. The results and general method of this paper conform to a section of the author's doctoral thesis, the research for which was carried on under the direction of Dr. Ernst Weber at the Polytechnic Institute of Brooklyn.

1J. C. Slater, Microwave transmission, McGraw Hill, 1942, pp. 71-74.

${ }^{2}$ Publication P. B. 6588 (U. S. Commerce Dept.), 1945. 
2. Differential equation of the problem. The voltage and current distribution in a co-axial line can be written as a pair of differential equations ${ }^{3}$

$$
d V / d x=I Z, \quad d I / d x=-V Y,
$$

where $V$ and $I$ are the voltage and current at any point $x$ on the transmission line, and $Z$ and $Y$ are the series impedance and shunt admittance per unit length at any

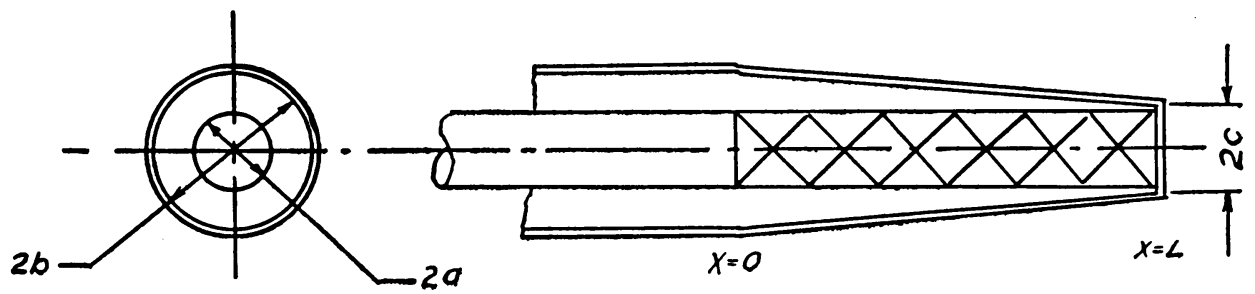

FIG. 1.

point $x$. Differentiating each of Eqs. (1) and combining, we obtain a pair of second order differential equations

$$
\begin{aligned}
& d^{2} I / d x^{2}-\left(Y^{\prime} / Y\right) d I / d x-Z Y I=0, \\
& d^{2} V / d x^{2}-\left(Z^{\prime} / Z\right) d V / d x-Z Y V=0,
\end{aligned}
$$

where $Y^{\prime}$ and $Z^{\prime}$ are the derivatives of $Y$ and $Z$ with respect to $x$. In general, with air filling the space between the conductors, we find that

$$
\begin{aligned}
& Z=R+i \omega k_{L} \log \left(r_{0} / r_{1}\right), \\
& Y=g+i \frac{\omega k_{c}}{\log \left(r_{0} / r_{1}\right)},
\end{aligned}
$$

where $R=$ resistance per unit length, $g=$ conductance per unit length, $\omega=2 \pi f, f=$ frequency, $k_{c}=2 \pi \epsilon_{0}=10^{-9} / 18$ farads $/$ meter, $k_{L}=\mu_{0} / 2 \pi=2 \times 10^{-7}$ henries $/$ meter, $r_{0}=$ radius of the outer conductor between $x=0$ and $x=L, r_{i}=$ radius of the inner conductor between $x=0$ and $x=L$.

The actual linear tapered line shown in Fig. 1 is now approximated by an exponential line in which the variation of the outer conductor is given by

$$
r_{0}=a(b / a) \exp (-k x) .
$$

We then find that (assuming $g=0, R$ constant)

$$
\begin{array}{ll}
Z=R+Z_{0} \exp (-k x), & Z_{0}=i \omega k_{L} \log (b / a), \\
Y=Y_{0} \exp (k x), & Y_{0}=i \omega k_{c} / \log (b / a),
\end{array}
$$

where $Z_{0}$ and $Y_{0}$ are, respectively, the series impedance and shunt admittance per unit length of a lossless uniform line $(R=g=0)$ having a constant outer radius $b$ and constant inner radius $a$. The differential equation (2) for $I$ will then become

$$
d^{2} I / d x^{2}-k d I / d x-\left[Y_{0} Z_{0}+R Y_{0} \exp (k x)\right] I=0 .
$$

3J. C. Slater, loc. cit., pp. 60-71. 
If we let

$$
s=\left[-4 R Y_{0} \exp (k x) / k^{2}\right]^{1 / 2}
$$

and set $I(x)=s W(s)$, we can transform Eq. (2) into

$$
d^{2} W / d s^{2}+(1 / s) d W / d s+\left[1-\left(4 Y_{0} Z_{0} / k^{2}\right)\left(1 / s^{2}\right)\right] W=0 .
$$

This differential equation for $W(s)$ is the Bessel equation of order

$$
\nu_{1,2}= \pm\left(4 Z_{0} Y_{0} / k^{2}\right)^{1 / 2} \text {. }
$$

Hence, we may write

$$
I(x)=\exp (k x / 2)\left[M J_{v_{1}}(s)+N J_{v_{\mathbf{s}}}(s)\right] .
$$

It should be pointed out that the substitution, Eq. (7), which resulted in the differential equation (8) was obtained only after a series solution of an integral equation was found.

3. Integral equation. The method of solution for the lossy exponential line by means of an integral equation was suggested by a "perturbation method" of Schelkunoff."

If we consider a lossless $(R=g=0)$ exponential line, then

$$
Y=Y_{0} \exp (k x), \quad Z=Z_{0} \exp (-k x),
$$

and Eq. (2) becomes

$$
d^{2} I / d x^{2}-k d I / d x-Z_{0} Y_{0} I=0 .
$$

Since $k$ and $Z_{0} Y_{0}$ are constants, the solution for $I(x)$ is

$$
I(x)=A \exp \left(m_{1} x\right)+B \exp \left(m_{2} x\right),
$$

where

$$
\begin{aligned}
& m_{1}=1 / 2\left[k+\left(k^{2}+4 \Gamma_{0}^{2}\right)^{1 / 2}\right], \\
& m_{2}=1 / 2\left[k-\left(k^{2}+4 \Gamma_{0}^{2}\right)^{1 / 2}\right],
\end{aligned}
$$

Using Eq. (1), we find that

$$
V(x)=-Y_{0}^{-1} \exp (-k x)\left[m_{1} A \exp \left(m_{1} x\right)+m_{2} B \exp \left(m_{2} x\right)\right] .
$$

We now assume that at $x=\epsilon$, there is a point discontinuity of $R d \epsilon$ ohms in series with the line. To the left of $x=\epsilon$

$$
\begin{aligned}
I_{0}(x) & =A \exp \left(m_{1} x\right)+B \exp \left(m_{2} x\right) \\
V_{0}(x) & =-Y_{0}^{-1} \exp (-k x)\left[A m_{1} \exp \left(m_{1} x\right)+B m_{2} \exp \left(m_{2} x\right)\right]
\end{aligned}
$$

To the right of $x=\epsilon$

$$
\begin{aligned}
I_{1}(x) & =C \exp \left(m_{1} x\right)+D \exp \left(m_{2} x\right) \\
V_{1}(x) & =-Y_{0}^{-1} \exp (-k x)\left[m_{1} C \exp \left(m_{1} x\right)+m_{2} D \exp \left(m_{2} x\right)\right]
\end{aligned}
$$

${ }^{4}$ S. A. Schelkunoff, Electromagnetic waves, D. Van Nostrand Co., 1943, ch. 4. 
At $x=\epsilon$, the boundary conditions for $V$ and $I$ become

$$
\begin{aligned}
I_{0}(\epsilon-0) & =I_{1}(\epsilon+0), \\
V_{0}(\epsilon-0) & =V_{1}(\epsilon+0)+V(\epsilon),
\end{aligned}
$$

where $V(\epsilon)=I(\epsilon) R d \epsilon$. We may now determine the constants $C$ and $D$ in terms of $A$ and $B$. If we assume an infinite distribution of discontinuities along the line, we obtain the following integral equation for $I$ :

$$
\begin{aligned}
I(x)= & I_{0}(x)-R Y_{0}\left(m_{2}-m_{1}\right)^{-1} \int_{0}^{x} I(\epsilon) \exp (k \epsilon) \\
\cdot & \left\{\exp \left[m_{1}(x-\epsilon)\right]-\exp \left[m_{2}(x-\epsilon)\right]\right\} d \epsilon .
\end{aligned}
$$

In Eq. (17), $R$ is constant.

Using Picard's method of successive substitution ${ }^{5}$ and rearranging terms, we find the infinite series

$$
\begin{aligned}
I(x)=E \exp \left(m_{1} x\right) & {\left[1+\frac{P \exp (k x)}{2 m_{1} k}+\frac{P^{2} \exp (2 k x)}{4 m_{1} k^{2}\left(k+2 m_{1}\right)}\right.} \\
& \left.+\frac{P^{3} \exp (3 k x)}{12 m_{1} k^{3}\left(k+2 m_{1}\right)\left(2 k+2 m_{1}\right)}+\cdots\right] \\
& +F \exp \left(m_{2} x\right)\left[1+\frac{P \exp (k x)}{2 m_{2} k}+\frac{P^{2} \exp (2 k x)}{4 m_{2} k^{3}\left(k+2 m_{2}\right)}+\cdots\right],
\end{aligned}
$$

where $E$ and $F$ are constants and $P=R Y_{0}$. With a further rearrangement of the series and a change of variables we obtain the Bessel solution of Eq. (10).

4. Comparison of exponential and linear tapered lines. In order to compare the exponential and the linear tapered lines, we must find the input impedance to the exponential line. This is obtained by using Eq. (1) and finding the quotient $V(x) / I(x)$ at $x=0$. From Eq. (10) we find that

$$
\begin{array}{r}
V(x)=(-1 / Y)\left\{M \exp (k x / 2)\left[(k / 2) J_{\nu_{1}}(s)+J_{\nu_{1}}^{\prime}(s)(d s / d x)\right]\right. \\
\left.+N \exp (k x / 2)\left[(k / 2) J_{\nu_{2}}(s)+J_{\nu_{2}}^{\prime}(s)(d s / d x)\right]\right\} .
\end{array}
$$

Applying the recurrence formulas for Bessel functions, ${ }^{6}$ and using the relation (Eq. (7)) $d s / d x=k s / 2$, we find that

$$
\begin{gathered}
V(x)=[-k \exp (k x / 2) / 2 Y]\left\{M\left[\left(\nu_{1}+1\right) J_{\nu_{1}}(s)-s J_{\nu_{1}+1}(s)\right]\right. \\
\left.+N\left[\left(\nu_{2}+1\right) J_{\nu_{2}}(s)-s J_{\nu_{2}+1}(s)\right]\right\} .
\end{gathered}
$$

Since the transmission line was shorted at $x=L$, we find that $V(L)=0$, and from Eq. (20) we have that

${ }^{5}$ E. G. Keller, Mathematics of modern engineering, vol. 2, John Wiley, 1942.

${ }^{6}$ N. W. McLachlan, Bessel functions for engineers, Oxford Univ. Press, 1941, p. 158. 


$$
\begin{gathered}
M / N=-\left[\left(\nu_{2}+1\right) J_{\nu_{2}}\left(s_{L}\right)-s_{L} J_{\nu_{2}+1}\left(s_{L}\right)\right] /\left[\left(\nu_{1}+1\right) J_{\nu_{1}}\left(s_{L}\right)-s_{L} J_{\nu_{1}+1}\left(s_{L}\right)\right], \\
s_{L}=\left[-4 R Y_{0} k^{-2} \exp (k L)\right]^{1 / 2} .
\end{gathered}
$$

Then the input impedance becomes

$$
Z_{\text {in }}=V(0) / I(0)=T(1-U) /(1-W),
$$

where

$$
\begin{aligned}
T & =-\frac{k}{2 Y_{0}}\left[\frac{\left(\nu_{2}+1\right) J_{\nu_{2}}\left(s_{0}\right)-s_{0} J_{\nu_{2}+1}\left(s_{0}\right)}{J_{\nu_{2}}\left(s_{0}\right)}\right], \\
U & =\frac{\left[\left(\nu_{2}+1\right) J_{\nu_{2}}\left(s_{L}\right)-s_{L} J_{\nu_{2}+1}\left(s_{L}\right)\right]\left[\left(\nu_{1}+1\right) J_{\nu_{1}}\left(s_{0}\right)-s_{0} J_{\nu_{1}+1}\left(s_{0}\right)\right]}{\left[\left(\nu_{1}+1\right) J_{\nu_{1}}\left(s_{L}\right)-s_{L} J_{\nu_{1}+1}\left(s_{L}\right)\right]\left[\left(\nu_{2}+1\right) J_{\nu_{2}}\left(s_{0}\right)-s_{0} J_{\nu_{2}+1}\left(s_{0}\right)\right]}, \\
W & =\frac{\left[\left(\nu_{2}+1\right) J_{\nu_{2}}\left(s_{L}\right)-s_{L} J_{\nu_{2}+1}\left(s_{L}\right)\right] J_{\nu_{1}}\left(s_{0}\right)}{\left[\left(\nu_{1}+1\right) J_{\nu_{1}}\left(s_{L}\right)-s_{L} J_{\nu_{1}+1}\left(s_{L}\right)\right] J_{\nu_{2}}\left(s_{0}\right)} .
\end{aligned}
$$

A measure of the input impedance of a termination is the voltage standing wave ratio $^{7}$ (VSWR) produced on the concentric co-axial line to which the impedance is attached. The VSWR is the ratio of the maximum voltage to the minimum voltage between the inner and outer conductors. If the impedance given by Eq. (22) is the terminating impedance on a transmission line having a characteristic impedance $Z_{c}$, then as shown by Slater, ${ }^{3}$ the VSWR produced by this termination is given by

$$
\begin{aligned}
\operatorname{VSWR} & =(1+|K|) /(1-|K|), \\
K & =\left(Z_{c}-Z_{\text {in }}\right) /\left(Z_{c}+Z_{\text {in }}\right) .
\end{aligned}
$$

In general, $Z_{c}, Z_{\mathrm{in}}$, and $K$ are complex quantities. In order to check the approximating exponential termination against the linear tapered termination, it would be necessary to calculate values for Bessel functions of complex argument and indices. At present there are no such tables available. Asymptotic expansions in the literature as well as those mentioned in Watson's treatise on Bessel functions ${ }^{8}$ are either not applicable or involve series which are no more rapidly convergent than the actual series themselves. Since we wish to determine how the input VSWR varies with the wave length of the impressed frequency, it is necessary to modify Eq. (22) in terms of $\lambda$, the wave length. Since

$$
\Gamma_{0}^{2}=Z_{0} Y_{0}=-(2 \pi)^{2} f^{2} \mu_{0} \epsilon_{0}=-(2 \pi)^{2} f^{2} v^{2}=-(2 \pi / \lambda)^{2},
$$

we find from Eqs. (7) and (9) that

$$
\begin{aligned}
\nu_{1,2} & = \pm i\left[(4 \pi / k \lambda)^{2}-1\right]^{1 / 2}, \\
s & =(2 / k) \exp (i \pi / 4)\left[2 \pi R \exp (k x) / \lambda Z_{c}\right]^{1 / 2} .
\end{aligned}
$$

${ }^{7}$ J. C. Slater, loc. cit., pp. 48-54 and King, Mimno, Wing, Transmission lines, antennas, and wave guides, McGraw-Hill, 1945, ch. I.

${ }^{8} \mathrm{G}$. N. Watson, Treatise on the theory of Bessel functions, Cambridge Press, 1945. 
The numerical check is made against the linear tapered line having a length of 5.5 centimeters and a total resistance of 59 ohms. This termination is connected to a coaxial line having a characteristic impedance of $46.4 \mathrm{ohms}$. ( $\left.a=0.406^{\prime \prime}, b=0.1875^{\prime \prime}\right)$. If $k$ is equal to $1.3 / L$, a comparison of the radii of the tapered outer conductors is given in Table 1.

TABLE 1

Radius of Outer Conductor

$\begin{array}{lcc}x / L & \text { Linear Taper } & \text { Exponential Taper } \\ 0.0 & .4067 \text { in. } & .4067 \text { in. } \\ 0.1 & .385 & .370 \\ 0.2 & .364 & .342 \\ 0.3 & .342 & .317 \\ 0.4 & .321 & .301 \\ 0.5 & .300 & .282 \\ 0.6 & .278 & .269 \\ 0.7 & .257 & .256 \\ 0.8 & .236 & .246 \\ 0.9 & .214 & .238 \\ 1.0 & .193 & .232\end{array}$

If in Eq. (24) we use the following values:

$$
\begin{aligned}
k & =1.3 / 0.055=23.636 \\
R & =59 / 0.055=1072.7 \mathrm{ohms} / \mathrm{meter}, \\
Z_{c} & =46.4 \mathrm{ohms},
\end{aligned}
$$

we will find the following comparison between the measured VSWR for the actual linear tapered line and the calculated VSWR for the exponential line.

TABLE 2

$\begin{array}{lccc}\lambda(\text { meters }) & f \text { (megacycles) } & \begin{array}{c}\text { Measured VSWR } \\ \text { Linear Tapered Line }\end{array} & \begin{array}{c}\text { Calculated VSWR } \\ \text { Exponential Line }\end{array} \\ 0.303 & 990 & 1.11 & 1.26 \\ 0.2796 & 1073 & 1.12 & 1.29 \\ 0.2545 & 1179 & 1.12 & 1.31 \\ 0.2389 & 1256 & 1.12 & 1.33 \\ 0.2108 & 1423 & 1.14 & 1.33 \\ 0.1993 & 1505 & 1.17 & 1.27 \\ 0.1200 & 2500 & 1.13 & 1.26 \\ 0.1111 & 2700 & 1.12 & 1.19 \\ 0.1001 & 2997 & 1.12 & 1.18 \\ 0.0899 & 3337 & 1.11 & 1.16 \\ 0.0805 & 3728 & 1.08 & 1.13 \\ 0.0756 & 3968 & 1.09 & 1.14\end{array}$

5. Discussion of results and design factors. The results of Table 2 indicate a relatively flat response for the VSWR over a broad band of frequencies. The differences between the calculated and the measured values are due to the following reasons:

1. Since the exponential line cannot duplicate exactly the linear tapered line, we will of necessity have a slight discrepancy. Now it is much more difficult to machine a tube with an exponential variation for its inner radius than to machine a straight taper. 
Moreover, since the exponential taper indicates a conservative result, it would be more practical to use the linear taper.

2. An assumption was made in setting up the original differential equation. In a rigorous solution of the electromagnetic fields existing between a pair of concentric cylinders, ${ }^{9}$ only the principal mode will be transmitted if the wave length of the impressed field is greater than $2 \pi(b-a)$, where $b$ is the radius of the outer conductor and $a$ is the radius of the inner conductor. In the principal mode, the electric field lines are perpendicular to the inner and outer conductors, while the magnetic field lines are concentric circles about the axis of the inner conductor. When these conditions exist, the differential equations (1) apply. In the case of the tapered line, in order to satisfy rigorously the boundary conditions, other modes of propagation will have to exist. Fortunately, if the taper of the outer conductor is not too abrupt, it is possible to neglect the higher modes. Thus, this is another source of error between calculated and measured values.

3. The inner conductor of the lossy tapered line is a glass tube coated with a metallic film. In our solution, we neglected to take into account the fact that the electric field penetrates through the film. As shown by Griemsmann, ${ }^{10}$ for a uniform line, the effect of this field can be expressed as a correction factor in the characteristic impedance and the attenuating factor of the transmission line. He shows that the first order correction for the field inside the film leads to the following expressions for the characteristic impedance and the attenuation factor:

$$
\begin{gathered}
Z_{c}=R_{0}\left\{1-i X-\left[\left(K^{2}-1\right) / 4 \log K-1 / 2\right](\beta b X)^{2}\right\}^{1 / 2}, \\
\Gamma=i \beta\left\{1-i X-\left[\left(K^{2}-1\right) / 4 \log K-1 / 2\right](\beta b X)^{2}\right\}^{1 / 2}
\end{gathered}
$$

where $\beta=2 \pi / \lambda, X=R \lambda / 2 \pi R_{0}, K=a / b$, and $R=$ resistance per unit length, $\lambda=$ wave length, $R_{0}=$ characteristic impedance of the lossless uniform line, $b=$ radius of the inner conductor, $a=$ radius of the outer conductor. For the size of the transmission line and resistance used in this problem, the factor $\left[\left(K^{2}-1\right) / 4 \log K-1 / 2\right](\beta b X)^{2}$ can have a value of 0.05 at the lower frequencies but is negligible at the higher frequencies. The change in $Z_{c}$ and $\Gamma$ could be interpreted physically as a change in the size of the outer conductor; therefore, the values in Table 1, indicating the linear and exponential line outer conductor radii, may be separated more than indicated. Since this phenomenon is more evident at lower frequencies, it may account for the further discrepancy between calculated and measured values at lower frequencies than at higher frequencies.

In the design of a linear tapered line termination, a fairly flat and low VSWR can be obtained, if the following design constants are taken into account.

1. $k$ in the exponential of Eq. (4) has a value of $1.3 / L$, where $L$ is the length of the taper in meters.

2. In Eq. (22) if

$$
L / \lambda \geqq 0.62 \quad \text { and } \quad R_{t} \lambda / 2 \pi Z_{c} L \leqq 0.2 \text {, }
$$

${ }^{9} J$. C. Slater, loc. cit., p. 162.

${ }^{10}$ J. W. K. Griemsmann, Design of a lossy co-axial transmission line, Doctoral Thesis, Brooklyn Poly. Inst., June, 1946. 
where $L=$ length of the taper in meters, $\lambda=$ wave length in meters, $R_{t}=$ total resistance of the inner conductor $=R L, Z_{c}=$ characteristic impedance of the uniform line having an outer radius $b$ and an inner radius $a$ of the input of the tapered line, then

$$
T \cong Z_{c} \text {. }
$$

3. If $4 \pi / k \lambda \geqq 6$, we will find that the series for $U$ and $W$ can be approximated by exponentials. In addition, if $R[\exp (k L)-1] / k Z_{c} \geqq 3.5$, then

$$
0.96 \leqq(1-U) /(1-W) \leqq 1.04 .
$$

Since $k L=1.3$, and $\exp (k L)=3.667$, we find that $(1-U) /(1-W)$ is nearly unity if

$$
R_{t} / Z_{c} \geqq 1.7 \text {. }
$$

Thus it can be stated that to satisfy the lower frequency range with a low VSWR, the film length must be long $(L=0.6 \lambda)$, and the total resistance must be near the $Z_{c}$ for extremely low frequencies. In the upper range of frequencies, we should have a total resistance of 1.7 times the characteristic impedance $Z_{c}$. Thus we must conclude for practical reasons, that it would seem more advisable to use the tapered line for frequencies above 1000 megacycles. Of course, if the restriction on the VSWR could be lessened to permit a VSWR of 1.2 or 1.3, then a larger range of frequencies could be covered by the linear tapered line.

\section{CAPACITY OF A PAIR OF INSULATED WIRES*}

\section{By W. HOWARD WISE (Bell Telephone Laboratories)}

1. Introduction. This problem has been treated in elegant fashion by Craggs and Tranter. ${ }^{1,2,3}$ Their first two papers employ a conformal transformation of the free space; the third paper works with charge distributions. They end up with an infinite determinant of value zero in which the unknown capacity appears in one element, and conclude that "satisfactory numerical approximations can be obtained by keeping only the first few rows and columns". ${ }^{3}$

This note is written to remark that the end result of a straightforward attack with bi-polar coordinates is

$$
C=\epsilon_{0} / 4\left\{\log \frac{s}{\chi}+\frac{\epsilon_{0}}{\epsilon} \log \frac{\chi}{v}-\sum_{n=1}^{\infty} k_{n}\right\},
$$

where $C=$ capacity, $\epsilon_{0}=$ dielectric constant of air, or other material outside the jackets, $\epsilon=$ dielectric constant of jacket material, $s=$ interaxial separation, $\chi=$ outer

${ }^{*}$ Received Feb. 7, 1949.

1J. W. Craggs and C. J. Tranter, The capacity of twin cable, Q. Appl. Math. 3, 268-272 (1945).

${ }^{2}$ J. W. Craggs and C. J. Tranter, The capacity of twin cable-II, Q. Appl. Math. 3, 380-383 (1946.)

${ }^{3} \mathrm{~J}$. W. Craggs and C. J. Tranter, The capacity of two-dimensional systems of conductors and dielectrics with circular boundaries, Q. J. of Math. (Oxford) 17, 138-144 (1946). 